\title{
Nilpotent Locally Convex Lie Algebras and Lie Field Structures ${ }^{\star}$
}

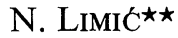 \\ Institute for Advanced Study, Princeton, New Jersey
}

Received March 8, 1969

\begin{abstract}
The purpose of this work is to join Lie field structures with certain infinitedimensional Lie algebras with locally convex topology. These topological Lie algebras allow topological groups which are a generalization of the connected nilpotent Lie groups. We showed the existence of the continuous unitary representations of the gained groups and then we proved the analogue of Gårding theorem. Using this theorem we established the existence of representations of Lie field structures into Lie algebras of skew-symmetric operators on Hilbert spaces.
\end{abstract}

\section{Introduction}

Infinite-dimensional Lie algebras with topological structures were already the object of the investigation. The semisimple Lie algebras with the topology of the Hilbert space and a particular property of the composition rule were considered by Schue $[17,18]$ and by Balachandran [1]. Also the infinite-dimensional Filtred Lie algebras with a topological structure were regarded by Veisfeiler [19]. Our aim is to concentrate on the infinite-dimensional Lie algebras with the locally convex topological structures which are the analogue of the nilpotent finite-dimensional Lie algebras and to look for possible groups related with these algebras.

The work is divided into sections with the following content. Definitions of different classes of the locally convex Lie algebras the analogue of which in the case of the finite-dimensional Lie algebras are always the nilpotent Lie algebras are given in Sections 1,2, 3, and 4. We have shown in Sections 5 and 6 the relation between certain topological groups and the locally convex Lie algebras of Section 3. A construction of the groups is offered in Section 5 and the connection of the unitary representations of the groups and the representations of the related locally convex Lie algebras is considered in Section 6. We proved an analogue of the results of Gårding [8] which is proved for the locally compact groups. In Section 7 we indicated the possible application of the developed theory in some problems of theoretical physics.

$\star$ Work supported by National Science Foundation.

$\star \star$ On leave of absence from the Institute "Rudjer Bošković", Zagreb.

7 Commun. math. Phys., Vol 14 


\section{Definition of Locally Convex Lie Algebra}

1.1. A 1.c. (locally convex) space over the field $K$ of real or complex numbers will be denoted by capital letters like $X, E, \ldots$, and its elements by small letters like $x, y, u, v, \ldots$ The base of absorbing, absolutely convex and balanced neighbourhoods of zero of a 1.c. space $X$ will be denoted by $\mathscr{U}(X)$. Elements of a 1.c. space will be called vectors when only the property of the 1.c. space is meant irrespectively on its possible other algebraic structure.

An abstract Lie algebra over $K$ is the object consisting of a vector space $X$ over $K$ and a mapping $L: X \times X \ni(x, y) \rightarrow L(x, y) \in X$, where $L(x, y)$ is $K$-linear function in every variable, satisfying two conditions

$$
L(x, x)=0, \quad L(x, L(y, z))+L(y, L(z, x))+L(z, L(x, y))=0,
$$

$x, y, z \in X$. The second condition is called Jacobi identity.

1.3. Definition. A 1.c. Lie algebra $\{X, \tau\}$ over $K$ is the abstract Lie algebra $X$ over $K$ and the complete 1.c. space $X$ over $K$ with the 1.c. topology $\tau$, such that the mapping $L$ is continuous in the first variable for any fixed second variable.

In the sequel we shall write $X$ instead of $\{X, \tau\}$ if such shorthand does not cause any misunderstanding. For two l.c. spaces $\{X, \sigma\}$ and $\{X, \tau\}$ we shall write $\{X, \sigma\} \subset\{X, \tau\}$ and $\sigma>\tau$, if the $\sigma$-topology is finer than the $\tau$-topology.

\section{Simultaneous Continuity of Mapping $L$}

2.1. For the purpose of the Section 5, where the groups corresponding to certain 1.c. Lie algebras are considered, the simultaneous continuity in both the variables of the mapping $L$ of 1.1 from $X \times X$ in $X$ is needed. If the mapping $L$ is not simultaneously continuous in both the variables for a particular l.c. Lie algebra $\{X, \tau\}$, we have a subsidiary task consisting in looking for some other topology $\sigma$ on $X$ such that the mapping $L$ would be continuous simultaneously in both the variables from $\{X, \sigma\}$ $\times\{X, \sigma\}$ into $\{X, \sigma\}$. We do not know how to obtain this other topology for the general l.c. Lie algebras and we can only tender a partial solution to this problem.

If $X$ is a metrizable 1.c. space then the mapping $L$ is simultaneously continuous in both the variables (Bourbaki [4], $\S 4$, No. 1). In this case the tensor produce $X \otimes X$ can be endowed with such topology $\omega$, that the canonical imbedding of the bilinear mapping $L$ from $X \times X$ into $X$ to the linear mapping from $\{X \otimes X, \omega\}$ into $\{X, \tau\}$ is continuous (Grothendieck [12]). It follows in particular that for every $U \in \mathscr{U}(X)$, there are $V_{1}, V_{2} \in \mathscr{U}(X)$ such that $p_{U}(L(x, y)) \leqq p_{V_{1}}(x) p_{V_{2}}(y)$, where $p_{W}$ is the 
Minkowski functional of $W \in \mathscr{U}(X)$. The right-hand side of the inequality can be made symmetrical by use of the semi-norm $p_{V}$ defined by $p_{V}(\mathrm{x})$ $=\sup \left\{p_{V_{1}}(x), p_{V_{2}}(x)\right\}$. Therefore we shall always have for the metrizable 1.c. Lie algebras

$$
p_{U}(L(x, y)) \leqq p_{V}(x) p_{V}(y)
$$

2.2. Let $\{X, \tau\}$ be the 1.c. Lie algebra and $\left\{A_{\alpha}, A\right\}$ a family of homomorphisms from $X$ into the 1.c. metrizable Lie algebras $Y_{\alpha}$ such that for every $x \in X, x \neq 0$, we have an $\alpha \in A$ with $A_{\alpha} x \neq 0$. The linear space $X$ furnished with the topology $\tau(K)$ of the locally convex kernel of the spaces $Y_{\alpha}$ is a l.c. space $\{X, \tau(K)\}$ (see Köthe [14]). It is an immediate consequence of the construction and of 2.1. that the induced mapping $L$ from $\{X, \tau(K)\}$ $\times\{X, \tau(K)\}$ into $\{X, \tau(K)\}$ by the mapping $L$ from $\{X, \tau\} \times\{X, \tau\}$ into $\{X, \tau\}$ is simultaneously continuous in both the variables, if for every $\alpha \in A$ only a finite number of elements $\alpha_{1}, \alpha_{2}, \ldots, \alpha_{n} \in A$ exists such that $L\left(Y_{\alpha_{1}}, Y_{\alpha_{j}}\right) \cap Y_{\alpha} \neq\{0\}$.

\section{Classes of Complex Nilpotent Locally Convex Lie Algebras}

3.1. For the subsets $Y, Z$ of the 1.c. Lie algebra $X$, we denote by $L(Y, Z)$ that subset of $X$ consisting of all the elements of $X$ which are of the form $L(y, z), y \in Y, z \in Z$. A subalgebra $Y$ of the l.c. Lie algebra $X$ is the subspace $Y$ of the space $X$ such that $L(Y, Y) \subseteq Y$. A subalgebra $Y$ is called closed if the subspace $Y$ is closed in the space $X$.

Lemma. Every commutative subalgebra $A$ of the l.c. Lie algebra $X$ can be imbedded into the closed commutative subalgebra $\bar{A}$, where the closed subspace $\bar{A}$ is the closure of the space $A$.

Proof. Let $A$ be a commutative subalgebra of the l.c. Lie algebra $X$. Then $L\left(a_{1}, a_{2}\right)-L\left(a_{2}, a_{1}\right)=0, a_{1}, a_{2} \in A$. On the other hand if $A$ is a subspace of the 1.c. space $X$ such that $L\left(a_{1}, a_{2}\right)-L\left(a_{2}, a_{1}\right)=0, a_{1}, a_{2} \in A$, then because of (1.2) we have $L\left(a_{1}, a_{2}\right)=0$ for any $a_{1}, a_{2} \in A$, i.e. $A$ is the commutative subalgebra of the 1.c. Lie algebra $X$. Hence to prove the Lemma one has to show that $L\left(a_{1}, a_{2}\right)-L\left(a_{2}, a_{1}\right)=0$ also for $a_{1}, a_{2} \in \bar{A}$. This can be done as for any topological algebra.

3.2. The normalizer $B$ of a subalgebra $A$ of the 1.c. Lie algebra $X$ is the set of all the elements $b \in X$ for which $L(b, a) \in A$ for any $a \in A$. The normalizer $B$ of a subalgebra $A$ is again the subalgebra. The subalgebra $A$ is called the ideal of the 1.c. Lie algebra $X$ if $X$ itself is the normalizer of $A$.

Lemma. The normalizer $B$ of a closed subalgebra $A$ is the closed subalgebra. For every ideal $A$ of $X$, the closed subspace $\bar{A}$ is also an ideal of $X$. 
Proof. For the closed subalgebra $A$ the sets $B^{\prime}(a)=(\operatorname{ad} a)^{-1}(A), a$ fixed, are the closed subalgebras containing the algebra $B$. The intersection $B^{\prime}=\bigcap_{a \in A} B^{\prime}(a)$ is the closed subalgebra which contains the algebra $B$ and $B^{\prime}$ is the normalizer of $A$. Because of the maximality of $B$ it holds $B=\bar{B}=B^{\prime}$. For the proof of the other part we notice that $\operatorname{ad} x(\bar{A}) \subset \bar{A}$. As $X \supset \bar{A}$ it follows that $L(\bar{A}, \bar{A}) \subset \bar{A}$, so that $\bar{A}$ is the subalgebra. Evidently $\bar{A}$ is the ideal of $X$.

3.3. Let $A_{1}$ and $A_{2}$ be subalgebras of $X$ where $A_{2} \supset A_{1}$. The subnormalizer $B$ of the algebras $A_{1}, A_{2}$, is the set of all the elements $b \in X$ for which $L\left(B, A_{2}\right) \subseteq A_{1}$. It is easy to prove as in the last two similar statements:

Lemma. The subnormalizer of two closed subalgebras $A_{1}, A_{2}, A_{1} \subset A_{2}$, is also the closed subalgebra.

The subnormalizer of the pair $A_{1}=\{0\}, A_{2}$ is called the centralizer.

3.4. Let $X$ be the 1.c. Lie algebra. Denote by $X^{k}$ the closure of the set of all the elements of the form $L(y, x), y \in X^{k-1}, x \in X$, and $X^{1}=X$. Thus $X^{k}=\overline{L\left(X^{k-1}, X\right)}$.

If $X$ is a finite-dimensional Lie algebra for which $X^{n}=\{0\}$ for some natural number $n$ we call this algebra nilpotent. Let us take now any subalgebra $X_{0} \subset Z$, where $Z$ is the center of the nilpotent Lie algebra $X$ and construct the subnormalizer $X_{1}$ for the pair $X_{0}, X$. The algebra $X_{1}$ is an ideal of $X$ containing $X_{0}$. Extending this prescript we come to a sequence $X_{0} \subseteq X_{1} \subseteq X_{2} \subseteq \cdots \subseteq X_{n}$. For the nilpotentalgebras $X$ we have $X_{n}=X$ for a natural number $n$ and therefore the finite-dimensional Lie algebra $X$ is nilpotent if and only if it has a finite chain of ideals $X_{0} \subset X_{1} \subset \cdots \subset X_{n}=X$, where $X_{0}$ is the center of $X$ and $X_{k}$ is the subnormalizer of the pair $X_{k-1}, X$. This nice situation brakes off when we go to the infinite-dimensional 1.c. Lie algebras. In particular there are 1.c. Lie algebras for which $\lim _{n \rightarrow \infty} X^{n}=\lim _{n \rightarrow \infty} \bigcap_{k=1}^{n} X^{k}=\{0\}$, although $X$ has no center. An illustration of this mischief gives the following example.

Let $X$ be the set of all the elements $x=\left\{x_{i k} \mid x_{i k}=0\right.$ for $\left.i \geqq k, i, k=1,2, \ldots\right\}$ and $x_{i k}$ are complex numbers. Hence $X$ is the set of all triangle matrices with the vanishing diagonal elements. $X$ is the linear space if the operations of the linear space are defined as usually with matrices. Let us introduce the norm on $X$ by the non-negative function $X \ni x \rightarrow\|x\|$ $=2\left(\sum_{i, k=1}^{\infty}\left|x_{i k}\right|^{2}\right)^{\frac{1}{2}} \in R_{+}$. The algebraic operation

$$
L(x, y)=\left\{\sum_{k=1}^{\infty}\left(x_{i k} y_{k j}-y_{i k} x_{k j}\right) \mid i, j=1,2, \ldots\right\}
$$


mapps $X \times X$ into $X$ continuously simultaneously in both the variables as $\|L(x, y)\| \leqq\|x\|\|y\|$. In this way we formed a l.c. Lie algebra. The elements $x^{(n, m)}=\left\{\delta_{i n} \delta_{j m} \mid i, j=1,2, \ldots ; n, m\right.$ fixed $\left.n<m\right\}$ form a basis of the 1.c. Lie algebra $X$. There is no non-trivial central element $z \in X$, as the condition $L\left(x^{(n, m)}, z\right)=0$ for any pair $n, m, n<m$, tells us that $z_{i k}=0$ for all $i, k=1,2, \ldots, i<k$. The subalgebras $X^{k}=\overline{L\left(X, X^{k-1}\right)}, X^{1}=X$, form the properly decreasing sequence of the ideals of $X$. If $Y=\lim _{n \rightarrow \infty} \bigcap_{k=1}^{n} X^{k}$ were the ideal different from $\{0\}$, an element $y=\left\{y_{i j} \mid i, j=1,2, \ldots\right\}$, $\|y\|>0$, would belong to $Y$. But $\|y\|=0$ as $\|y\|^{2}=4 \sum_{i, j=1}^{\infty}\left|y_{i j}\right|^{2}$ and $y \in X^{k}$ for every $k$, i.e. $y_{i j}=0$ for every $i \geqq j+k, k=1,2, \ldots$.

This forces us to operate with different kinds of Lie algebras the analogue of which in the case of the finite-dimensional Lie algebras is always the same object - the nilpotent Lie algebra. Here we define two kinds of the nilpotent 1.c. Lie algebras which will be handled easily and usefully in our further investigations.

3.5. 'Let $X$ be the 1.c. Lie algebra with the center $Z$. The center is the closed ideal of $X$ because of its maximality and the Lemma 3.3. Denoting $X_{0}=Z$, we consider the sequence of ideals $X_{0} \subseteq X_{1} \subseteq \cdots$, where $X_{k}$ is the subnormalizer of the pair $X_{k-1}, X, k=1,2, \ldots$ All $X_{k}$ are the closed ideals according to the Lemma 3.3.

Definition. The properly nilpotent l.c. Lie algebra $X$ is such 1.c. Lie algebra $X$ for which a chain $X_{0} \subseteq X_{1} \subseteq X_{2} \subseteq \cdots$ exists, where $X_{0}$ is the center of $X$ and $X_{k}$ is the closed ideal and the subnormalizer of the pair $X_{k-1}, X, k=1,2, \ldots$, and $X=\bigcup_{n=0}^{\infty} X_{n}$. If $X=X_{n}$ for some natural number $n X$ is called the finitely nilpotent 1.c. Lie algebra if $L(X, X) \subset X_{n-1}$.

3.6. In accordance with 3.4 the next reasonable class of the nilpotent 1.c. Lie algebras is given by

Definition. A 1.c. Lie algebra $X$ for which $\lim _{n \rightarrow \infty} \bigcap_{k=1}^{n} X^{k}=\{0\}$, where $X^{k}=\overline{L\left(X^{k-1}, X\right)}, X^{1}=X$, is called the nilpotent 1.c. Lie algebra.

3.7. The Theorem of Engel states that a finite-dimensional Lie algebra $X$ is nilpotent if and only if ad $x$, for every $x \in X$, is the nilpotent operator on $X$. For the infinite-dimensional l.c. Lie algebras no analogous statement can be made in general. To see this we offer an example of a normal Lie algebra $X$ for which ad $x, x \in X$, is bounded, quasinilpotent operator on the Banach space $X$, but $X$ is neither the properlynilpotent nor nilpotent l.c. Lie algebra. This example shows in the same time that restricting our interest to the properly nilpotent and nilpotent 1.c. Lie algebras we occupy ourselves only with two classes of the l.c. Lie algebras 
the analogue of which in the finite-dimensional case is the nilpotent Lie algebra.

Let $I$ be the interval $[0,1]$ and $C(I \times I)$ the linear space of all the continuous functions on $I \times I$. The semi-norm $C(I \times I) \ni x \rightarrow\|x\|_{c} \in R_{+}$ defined by $\|x\|_{c}^{2}=\int|x(p, q)|^{2} d p d q$ gives the Hilbert space $L^{2}(I \times I)$ $=\overline{C(I \times I)} /\left\{x \in \overline{C(I \times I)} \mid\|x\|_{c}=0\right\}$ with the scalar product $\sigma(x, y)$. The structure of a Lie algebra on $L^{2}(I \times I)$ can be introduced by the following bilinear mapping

$$
\begin{aligned}
& L^{2}(I \times I) \times L^{2}(I \times I) \ni(x, y) \rightarrow L(x, y): \\
& \quad=\left\{\int_{I}[x(p, r) y(r, q)-y(p, r) x(r, q)] d r \mid p, q \in I\right\} \in L^{2}(I \times I) .
\end{aligned}
$$

It is easy to see that actually an abstract Lie algebra is gained in this way. This abstract Lie algebra is inverted to the normed Lie algebra by the norm $X \ni x \rightarrow\|x\|=2[\sigma(x, x)]^{\frac{1}{2}} \in R_{+}$. By the direct inspection one sees that $\|L(x, y)\| \leqq\|x\|\|y\|$ for every $x, y \in L^{2}(I \times I)$ and therefore $L^{2}(I \times I)$ is the complete normed Lie algebra. The subspace $X$ of the space $L^{2}(I \times I)$ determined by all the vectors $x \in L^{2}(I \times I)$ for which a representative $x(p, q)$ exists such that $x(p, q)=0$ if $p \leqq q$ gives us the complete normed Lie algebra $X$ already accomplished with all the desired properties.

Let $z,\|z\|=1$, be a central element of $X$ represented by the function $z(p, q)$. For any two closed intervals $A_{1}$ and $A_{3}$ of $(0,1)$ such that $A_{1}>A_{3}$ we shall denote by $A_{2}$ a closed interval between them and by $\chi_{23}(p, q)$ the characteristic function of $A_{2} \times A_{3} C I \times I$. Because of $L\left(z, \chi_{23}\right)(p, q)=0$ almost everywhere an $A_{1} \times A_{3}$, the function $\int_{A_{2}} z(p, r) d r$ of one variable $p$ almost everywhere vanishes on $A_{1}$. The intervals $A_{1}$ and $A_{3}$ are arbitrary such that $A_{1}>A_{3}$ and $z(p, q)=0$ almost everywhere on $\{p, q \mid p \leqq q\}$ $C I \times I$ so that $\int_{A \times B} z(p, q) d p d q=0$ for any two closed intervals $A, B \subset(0,1)$.

Hence $z(p, q)$ vanishes almost everywhere on $I \times I$ contrary to the supposition $\|z\|=1$. This means that $X$ cannot be the properly nilpotent normed Lie algebra.

Suppose now that $X$ is the nilpotent normed Lie algebra, i.e. $\overline{L(X, X)} \subset X$. For any three non-trivial closed intervals $A_{1}, A_{2}, A_{3}, A_{1} \geqq A_{2} \geqq A_{3}$, of the interval $(0,1)$ we consider two characteristic functions $\chi_{12}$ and $\chi_{23}$ of the sets $A_{1} \times A_{2}$ and $A_{2} \times A_{3}$ respectively. The function $L\left(\chi_{12}, \chi_{23}\right)(p, q)$ is proportional to the characteristic function of the set $A_{1} \times A_{3}$. In this way we can construe the characteristic function of any square $A \times B$ such that $\bar{A}>\bar{B}$ which contradicts the supposition $\overline{L(X, X)} \subset X$. Hence our algebra cannot be the nilpotent normed Lie algebra.

It remains to show that $\operatorname{ad} x, x \in X$, is the quasinilpotent operator on $X$. The vector $(\operatorname{ad} x)^{n} y$ of the space $X$ is the sum of $2^{n}$ vectors each 
having the following form

$$
z(p, q)=\int x_{\alpha_{1}}\left(p, r_{1}\right) d r_{1} x_{\alpha_{2}}\left(r_{1}, r_{2}\right) d r_{2} \ldots d r_{n} x_{\alpha_{n+1}}\left(r_{n}, q\right),
$$

where the integration is extended on the interval $\underbrace{I \times I \times \cdots \times I}_{n \text {-times }}$, $p \geqq r_{1} \geqq r_{2} \geqq \cdots \geqq r_{n} \geqq q$, and one of $x_{\alpha_{1}}, x_{\alpha_{2}}, \ldots, x_{\alpha_{n+1}}$ is equal to $y$ and all the others are equal to $x$. Thus we have $|z(p, q)| \leqq\|x\|^{n}\|y\| / 2^{n+1} \cdot n$ ! and therefore

$$
\left\|(\operatorname{ad} x)^{n} y\right\| \leqq\|y\| \frac{\|x\|^{n}}{n !} .
$$

It follows from the attained estimation that $\mathrm{ad} x$ is the quasinilpotent operator on $X$.

Let $X$ be a complex complete 1.c. space and $A$ a continuous operator from $X$ into $X$. If $A-\lambda I$ has the two-sided continuous inverse $(A-\lambda I)^{-1}$ for every complex $\lambda, \lambda \neq 0$, we shall say that $A$ is the quasinilpotent operator on $X$.

In view of the example we have to be aware of the existence of the objects defined in the following way.

Definition. The complex 1.c. Lie algebra $X$ for which ad $x, x \in X$, is the quasinilpotent operator is called the quasinilpotent l.c. Lie algebra.

\section{Relation between Complex and Real Lie Algebras}

4.1. A real finite-dimensional Lie algebra $X$ has the extension to the complex Lie algebra $X^{c}$ such that $\operatorname{dim}_{c} X^{c}=\operatorname{dim}_{R} X$. This extension $X^{c}$ is called the complexification of the real algebra $X$. We shall define in analogy with the finite-dimensional case the complexification of the 1.c. Lie algebra over the field of real numbers. The tensor product $X^{c}=C \otimes X$ is furnished by the topology of bi-equicontinuous convergence (Grothendieck [12], see also Pietsch [16]. In the latter reference the topology is named $\varepsilon$-topology). The mapping $C \times X^{c} \ni\left(\lambda, \sum \alpha_{k} x_{k}\right)$ $\rightarrow \sum \lambda \alpha_{k} x_{k} \in X^{c}$ is continuous so that $X$ after completionis the completel.c. space. If the mapping $L$ from $X \times X$ into $X$ is extended to the mapping $L$ from $\overline{X^{c}} \times \overline{X^{c}}$ into $\overline{X^{c}}$ by

$$
L(x, y)=\sum_{k, l} \alpha_{k} \beta_{l} L\left(x_{k}, y_{l}\right)
$$

for $x=\sum \alpha_{k} x_{k} \in \overline{X^{c}}$ and $y=\sum \beta_{l} y_{l} \in \overline{X^{c}}$, then we check easily that this mapping satisfies (1.2). The continuity of $L$ in the first variable for the fixed second variable is induced by the corresponding continuity from $X$ into $X$ and the definition (4.2). Hence $\overline{X^{c}}$ is the 1.c. Lie algebra. 
4.3. The real 1.c. Lie algebra $X$ is properly nilpotent or nilpotent if and only if its complexification is properly nilpotent or nilpotent respectively. This statement is easily verified. The l.c. Lie algebra over the field of real numbers is called quasinilpotent if its complexification is the quasinilpotent 1.c. Lie algebra.

\section{Topological Groups Allowed by the l.c. Lie Algebras}

5.1. We try now to associate certain groups to the algebras which were pondered in Section 3. We shall use the construction of the group proposed by Birkhoff [2] or Dynkin [7] lent on the Campbell-Hausdorff formula. Birkhoff and Dynkin construed the local topological group for every normed Lie algebra (finite-dimensional or infinite-dimensional as the construction is the same). We found that the Dynkin's method is the most appropriate one for our case. Even more, because of nilpotency in one or the other way of the algebras considered here, it is possible to construe the whole connected group but not the local one only.

The aim of this work is not to consider unitary representations of the constructed groups but only to use these representations in order to show that there are representations of certain classes of l.c. Lie algebras into a Lie algebra of operators on Hilbert spaces which are skew-symmetric on a common invariant domain. The lack of an invariant measure on the groups forced us to use the invariant means and invariant functionals. For the survey of the invariant means the modern book by Hewitt and Ross [13] would be of a significant help for an uninitiated reader.

5.2. We shall say that the 1.c. Lie algebra $\{X, \tau\}$ allows the topological group $G$ if the underlying space $G$ is homeomorphic to the 1.c. space $\{X, \gamma\},\{X, \gamma\} \cap\{X, \tau\}$ is a dense linear subspace of $\{X, \tau\}$, such that the unity $e \in G$ is mapped to $0 \in\{X, \gamma\}$ by this homeomorphism. Hence the induced mappings $\{X, \gamma\} \times\{X, \gamma\} \ni(x, y) \rightarrow x \circ y \in\{X, \gamma\}$ and $\{X, \gamma\}$ $\ni X \rightarrow x^{-1} \in\{X, \gamma\}$ are continuous in the $\gamma$-topology and the former one is continuous simultaneously in the both variables.

Lemma. The properly nilpotent l.c. Lie algebra $X=\bigcup_{n=0}^{\infty} X_{n}$, for which the mapping $L$ is simultaneously continuous in both the variables, allows the topological group $G$ the topological space of which is the strict inductive limes of $X_{n}, n=0,1,2, \ldots$.

$$
\text { Proof. Let } X=\bigcup_{n=0}^{\infty} X_{n} \text { be properly nilpotent 1.c. Lie algebra and } X_{n} \text { the }
$$

subnormalizer of the pair $X_{n-1}, X, n=1,2, \ldots, X_{0}$ the center of $X . X_{n}$ is the ideal of $X$ and we can easily construct the group allowed by $X_{n}$. 
Let us remind that ad $x_{1} \operatorname{ad} x_{2} \ldots$ ad $x_{n-1}\left(x_{n}\right)=0$ for any $n$ elements $x_{1}, x_{2}, \ldots, x_{n} \in X_{n}$. By the Campbell-Hausdorff formula for any two $x, y \in X_{n}$ we have

$$
\begin{aligned}
& z=x \circ y=\sum_{m=1}^{\infty} \sum_{p_{1}, q_{1}, \ldots, q_{m}} \frac{(-)^{m}}{m} \frac{1}{\sum\left(p_{i}+q_{i}\right)} \frac{1}{p_{1} ! q_{1} ! \ldots q_{m} !}
\end{aligned}
$$

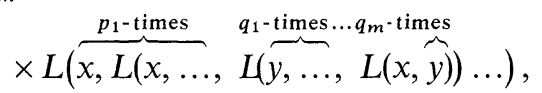

where $p_{i}+q_{i}>0, i=1,2, \ldots, m ; q_{m}=0$ or $q_{m}=1$. Because of $p_{i}+q_{i}>0$, $i=1,2, \ldots, m$, and the nilpotency of $X_{n}$ only the finite number of terms, depending on $n$ only, is different from zero in (5.3). We shall show now that for every neighbourhood $U \in \mathscr{U}(X)$ a neighbourhood $V \in \mathscr{U}(X)$ exists such that $V \times V \ni(x, y) \rightarrow x \circ y \in U$. According to the supposition on $L$ for every neighbourhood $U_{1} \in \mathscr{U}(X)$, there is a neighbourhood $U_{2} \in \mathscr{U}(X)$ such that the corresponding Minkowski functionals $p_{U_{1}}, p_{U_{2}}$ satisfy $p_{U_{1}}(L(x, y)) \leqq p_{U_{2}}(x) p_{U_{2}}(y)$. For the argumentation see 2.1 . In this way we choose a sequence of the neighbourhoods $U=U_{1}, U_{2}, \ldots, U_{n}$, for which $p_{U_{k}}(L(x, y)) \leqq p_{U_{k+1}}(x) p_{U_{k+1}}(y), k=1,2, \ldots, n-1$. Now we have the estimate

$$
\begin{aligned}
& p_{U}(x \circ y) \leqq \sum_{m=1}^{\infty} \sum_{p_{1}, q_{1}, \ldots, q_{m}} \frac{1}{m} \frac{1}{\sum\left(p_{i}+q_{i}\right)} \frac{1}{p_{1} ! q_{1} ! \ldots q_{m} !} \\
& p_{U_{2}}(x) p_{U_{3}}(x) \ldots p_{U_{l}}(y),
\end{aligned}
$$

where the index $l$ has the value $\sum\left(p_{i}+q_{i}\right)$. Because of $p_{U_{k}} \leqq p_{U_{k+1}}$ in our construction we can estimate this inequality as

$$
p_{U}(x \circ y) \leqq e^{2 n} \max \left\{p_{U_{n}}(x)^{n-2}, p_{U_{n}}(y)^{n-2}\right\}\left(p_{U_{n}}(x)+p_{U_{n}}(y)\right) .
$$

If $V=e^{-2 n} U_{n}$ then for every pair $x, y \in V$ we have $x \circ y \in U$ what we wished to prove. It is not difficult to see that $(x \circ y) \circ z=x \circ(y \circ z)$, $x \circ(-x)=0$. This enables us to recognize the following topological group:

The 1.c. space $X_{n}$ is the underlying space of the topological group $G_{n}$ with the base $\mathscr{U}\left(G_{n}\right)=\mathscr{U}\left(X_{n}\right)$ of the neighbourhoods of the unity $e \in G_{n}$, $e=0 \in X_{n}$, if the group operation is defined by (5.3) and the inverse element $g^{-1} \in G_{n}$ of the element $g \in G_{n}, g=x \in X_{n}$, is defined by $g^{-1}=-x \in X_{n}$. The mappings $G_{n} \times G_{n} \ni\left(g_{1}, g_{2}\right)=\left(x_{1}, x_{2}\right) \rightarrow x_{1} \circ x_{2}=g_{1} g_{2} \in G_{n}$ and $G_{n} \ni g=x \rightarrow-x=g^{-1} \in G_{n}$ are continuous.

We now have the sequence of the groups $G_{0} \leqq G_{1} \leqq G_{2} \leqq \cdots$ allowed by the 1.c. Lie algebras $X_{0}, X_{1}, X_{2}, \ldots$. We shall define a topological group $G$ allowed by the properly nilpotent l.c. Lie algebra $X=\bigcup_{n=0}^{\infty} X_{n}$ 
as the topological group $G=\bigcup_{n=0}^{\infty} G_{n}$ the underlying space of which is the strict inductive limes of $X_{n}, n=0,1,2, \ldots$. The strict inductive limes is possible as the topology of $X_{n-1}$ is the induced topology by $X_{n}, n=1,2, \ldots$. Even more the space $X=\operatorname{str} . \lim X_{n}$ is complete as $X_{n-1}$ is closed in $X_{n}, n=1,2, \ldots$. This finishes the proof.

It is not obvious that the abstract group $G$ becomes a topological group when furnished with the described topology. In general this statement is valid if $G_{0}, G_{1}, \ldots$, have special properties. In our case the 1.c. Lie algebras $X_{0}, X_{1}, \ldots$, are finitely nilpotent and the statement can be easily verified.

5.4. The nilpotent 1.c. Lie algebra $X, \bigcap_{m=1}^{\infty} X^{m}=\{0\}, X^{1}=X$, $X^{m}=\overline{L\left(X, X^{m-1}\right)}$, can be mapped into the finitely nilpotent 1.c. Lie algebra $Y_{m}=X / X^{m}$ in a natural way and the corresponding mapping $A_{m}$ is the continuous linear homomorphism from $X$ onto $Y_{m}$. Let us introduce the topology on the set $X$ by the kernel topology of the locally convex kernel $K_{m} A_{m}^{-1}\left(\left\{Y_{m}, \tau_{m}\right\}\right)$, where by $\tau_{m}$ is denoted the topology of the space $Y_{m}=X / X^{m}$ induced by the $\tau$-topology of the space $X$. The kernel topology will be denoted by $\tau(K)$. The neighbourhoods of zero of $\{X, \tau(K)\}$ are the sets $\left\{A_{1}^{-1}\left(U_{1}\right), A_{2}^{-1}\left(U_{2}\right), \ldots\right\}$, where $U_{k}$ are the neighbourhoods of zero of the spaces $Y_{k}$ for the finite number of indices $k=1,2, \ldots$, and for all other $k, U_{k}=Y_{k}$. In fact $\{X, \tau(K)\}$ is the topological projective limes of the complete 1.c. spaces $Y_{m}$. This results to the completeness of the space $\{X, \tau(K)\}$ (see for instance Köthe [14]).

Lemma. The nilpotent l.c. Lie algebra $X$, for which the mapping $L$ is simultaneously continuous in both the variables, allows the topological group $G$ the underlying space of which is $\{X, \tau(K)\}$.

Proof. For every neighbourhood $U=\left\{A_{1}^{-1}\left(U_{1}\right), A_{2}^{-1}\left(U_{2}\right), \ldots\right\}$ we consider the associated neighbourhoods $U_{1}, U_{2}, \ldots$, of the finitely nilpotent l.c. Lie algebras $Y_{1}, Y_{2}, \ldots$ By 5.2 a neighbourhood $V_{k}$ can be picked for every $Y_{k}, k=1,2, \ldots$, such that $V_{k} \times V_{k} \ni(x, y) \rightarrow x \circ y \in U_{k}$, i.e. the mapping $Y_{k} \times Y_{k} \quad(x, y) \rightarrow x \circ y \in Y_{k}$ is the continuous mapping. The mapping $Y_{k} \ni x \rightarrow-x \in Y_{k}$ is already continuous. Then for the neighbourhood $V=\left\{A_{1}^{-1}\left(V_{1}\right), A_{2}^{-1}\left(V_{2}\right), \ldots\right\}$, where $V_{k}=Y_{k}$ if $U_{k}=Y_{k}$, we have $V \times V \ni(x, y) \rightarrow x \circ y \in U$ and, of course, $U \ni x \rightarrow-x \in U$. We obtained the topological group of the Lemma.

5.5. Let $G$ be the topological group allowed by the 1.c. Lie algebra $X$. Then every subalgebra $Y$ of $X$ allows some subgroup $H$ of $G$ and every ideal $Y$ of $X$ allows some normal subgroup $H$ of $G$. These assertions are the quick consequences of the formula (5.3) which defines the group operation (see for instance Birkhoff [2] or Dynkin [7]). 
5.6. Let $G$ be the topological group and $X$ a real l.c. space $X$ with a basis $\left\{e_{k} \mid k=1,2, \ldots\right\}$. We shall say that the topological group $G$ admits a coordinate system $G \ni g \rightarrow T(g)=\left\{t_{k}(g) \mid k=1,2, \ldots\right\}$ if a homeomorphism $T$ from the underlying space of $G$ onto $X$ exists such that $T(g)$ $=\sum t_{k}(g) e_{k}$ for every $g \in G$ and $T(e)=0$ for the unit element $e \in G$. Hence the 1.c. space $X$ can be considered as the underlying space of the topological group $G$ where the group operation $X \times X \ni\left(x=\sum s_{k} e_{k}\right.$, $\left.y=\sum t_{k} e_{k}\right) \rightarrow \sum z_{k}\left(s_{1}, t_{1}, s_{2}, t_{2}, \ldots\right) e_{k} \in X$ is defined by the sequence of real functions $z_{1}, z_{2}, \ldots$, of real variables $s_{1}, t_{1}, s_{2}, t_{2}, \ldots$ The functions $z_{k}, k=1,2, \ldots$, receive finite values for those values of $s_{1}, t_{1}, s_{2}, t_{2}, \ldots$, for which $\sum s_{k} e_{k} \in X$ and $\sum t_{k} e_{k} \in X$.

Definition. The topological group $G$ for which three conditions listed below are satisfied is called the real entire Lie group.

1) Any two elements $g_{1}, g_{2} \in G$ can be placed in a subgroup $H \subset G$ admitting a coordinate system $H \ni h \rightarrow T(h)=\left\{t_{k}(h) \mid k=1,2, \ldots\right\}$.

2) If $T\left(g_{1}\right)=\sum s_{k} e_{k}$ and $T\left(g_{2}\right)=\sum t_{k} e_{k}, g_{1}, g_{2} \in H$, then $T\left(g_{1} g_{2}\right)$ $=\sum z_{k}\left(s_{1}, t_{1}, s_{2}, t_{2}, \ldots\right) e_{k}$ and $z_{k}\left(s_{1}, t_{1}, s_{2}, t_{2}, \ldots\right), k=1,2, \ldots$, are entire functions of any finite number of variables with all other variables fixed.

3) $z_{k}(0,0,0, \ldots)=0$ and $\frac{\partial}{\partial s_{k}} z_{l}(0,0,0, \ldots)=\frac{\partial}{\partial t_{k}} z_{l}(0,0,0, \ldots)=\delta_{k l}$.

5.7. Theorem. Every real properly nilpotent l.c. Lie algebra $X$ for which the mapping $L$ is simultaneously continuous in both the variables allows a real entire Lie group.

Proof. The Lemma 5.2 offers a topological group $G$ for every properly nilpotent 1.c. Lie algebra $\{X, \tau\}$ of the theorem. As the underlying space of $G$ is identified with the subspace $\{X, \gamma\}$ of the space $\{X, \tau\}$ the homeomorphism $T$ of 5.6 is trivial whenever the conditions 1) of 5.6 is fulfilled. Therefore, we write $x$ instead of $g$. Any two elements $x_{1}, x_{2} \in G \equiv\{X, \gamma\}$ are in some $G_{n} \equiv X_{n}$ as every bounded set of the strict inductive limes $X=\operatorname{str} . \lim X_{n}$, where $X_{n}$ is closed in $X_{n+1}, n=0,1,2, \ldots$, is contained in some $X_{n}$ and is bounded there (Dieudonné and Schwartz [5]). The elements $x_{1}, x_{2}$ of the Lie algebra $X_{n}$ generate a real nilpotent finitedimensional Lie algebra $Y$. Then the elements $x_{1}, x_{2}$ of the group $G_{n}=X_{n}$ can be placed in the real nilpotent finite-dimensional Lie subgroup $H$, the Lie algebra of which is $Y$. Thus the conditions $1-3$ of 5.6 are fulfilled. The theorem is proved.

5.8. Theorem. Every real nilpotent l.c. Lie algebra $X$ for which the mapping $L$ is simultaneously continuous in both the variables allows a real entire Lie group.

Proof. A topological group $G$ allowed by the nilpotent l.c. Lie algebra $X, \bigcap_{m=1}^{\infty} X^{m}=\{0\}$, is constructed in the Lemma 5.4. We shall show now that 
this group is actually the real entire Lie group. As in $5.7 T$ is trivial mapping so that for $g_{1}, g_{2} \in G$ we have $x_{1}=g_{1}, x_{2}=g_{2}, x_{1}, x_{2} \in\{X, \tau(K)\}$. The subalgebra $Y$ of the algebra $\{X, \tau(K)\}$ generated by two elements $x_{1}, x_{2} \in\{X, \tau(K)\}$ has a basis. We shall order this basis $\left\{e_{k} \mid k=1,2, \ldots\right\}$ such that $e_{k+1} \in X^{m}, m=n, n+1, \ldots$, if $e_{k} \in X^{n}$.

Let $x, y \in Y, x=\sum s_{k} e_{k}, y=\sum t_{k} e_{k}$. The continuous linear mapping $A_{m}$ of 5.4 maps $\{X, \tau(K)\}$ onto $\left\{X / X^{m}, \tau\right\}$. The subalgebra $Y$ is mapped to the nilpotent finite-dimensional Lie algebra $A_{m}(Y) \subset\left\{X / X^{m}, \tau\right\}$. Hence because of the special choice of the basis $\left\{e_{k} \mid k=1,2, \ldots\right\} \subset Y$, only a finite number $N_{k}$ of terms in the formula (5.3) contain the vector $e_{k}$ for a definite $k\left(N_{k+1} \geqq N_{k}\right)$. This implies that the function $z_{k}\left(s_{1}, t_{1}, s_{2}, t_{2}, \ldots\right)$ is a real polynimial in a finite number of variables so that the conditions of the Definition 5.6 are satisfied. The theorem is proved.

5.9. One-parameter subgroups of the entire Lie group $G$ are analytic homomorphisms from $R$ into $G$. The entire Lie groups of 5.7 and 5.8 have one-parameter subgroups of the form $g_{x}(t)=t x, x \in\{X, \gamma\}$ and they are the only ones. Even more for any two one-parameter subgroups $g_{x}(t)$ and $g_{y}(t)$ we have

$$
\lim _{t^{2} \rightarrow 0} \frac{1}{t^{2}} g_{x}(t) g_{y}(t) g_{x}(-t) g_{y}(-t)=L(x, y) .
$$

The formula (5.10) together with the shape of any one-parameter subgroup of the groups of 5.7 and 5.8 suggests to us to term the 1.c. Lie algebra $\{X, \gamma\}$ as the Lie algebra of the real entire Lie group of 5.7 and 5.8. We shall name in the following these two groups as the real properly nilpotent and nilpotent entire Lie group respectively.

Perhaps, it would be of interest to see whether every real entire Lie group $G$ has the Lie algebra, i.e. a real 1.c. Lie algebra $X$ and a homeomorphism $T$ from $G$ onto $X$ such that all the one-parameter subgroups have the form $g_{x}(t)=T^{-1}(t x), t \in R$, and

$$
\lim _{t^{2} \rightarrow 0} T\left(g_{x}(t) g_{y}(t) g_{x}(-t) g_{y}(-t)\right)=L(x, y) \in X,
$$

for any pair $x, y \in X$. However, this is out of the scope of our work as we are focused on the properly nilpotent and nilpotent 1.c. Lie algebras and their possible Lie groups.

\section{Invariant Functionals and Representations}

6.1. The space $C(G)$ of all the continuous functions on the topological group $G$ is a Banach space with the norm $C(G) f \rightarrow\|f\|_{G}$ $=\sup \{|f(g)| \mid g \in G\} \in R_{+}$. 
A function $f(g), f \in C(G)$, where $G$ is the real entire Lie group having Lie algebra $X$, is called infinitely differentiable in the $g$-direction if the function $f_{T}(t)=f\left(g_{x}(t)\right), g_{x}(t)=T^{-1}(t x), g(1)=g$, belongs to $C^{\infty}(R)$. A function $f(g), f \in C(G)$, will be called infinitely differentiable on the group $G$ if this function is infinitely differentiable in every g-direction, $g \in G$. The set of all the infinitely differentiable functions on the group $G$ span a linear subspace $C^{\infty}(G)$ of the space $C(G)$.

The linear space $C^{\infty}(G)$ is not empty for the real entire Lie group $G$ having Lie algebra $X$. Let $H$ be any subgroup of $G$ admitting the coordinate system related with the homeomorphism $T$ from $G$ onto $X$. The function $f(g)$ defined on $H$ by $f(g)=f_{T}\left(t_{1}, t_{2}, \ldots, t_{n}\right)$, where $T(g)=\sum_{k=1}^{n} t_{k} e_{k}+x$, and $f_{T}\left(t_{1}, t_{2}, \ldots, t_{n}\right) \in C^{\infty}\left(R_{n}\right)$, is certainly a vector of $C^{\infty}(H)$. Let us extend the domain of the function $f(g)$ from $H$ to the whole of $G$ setting $f(g)=f_{T}\left(t_{1}, t_{2}, \ldots, t_{n}\right)$ for $T(g)=\sum_{k=1}^{n} t_{k} e_{k}+x$, where $g, T^{-1}\left(e_{1}\right), \ldots, T^{-1}\left(e_{n}\right)$, belong to any subgroup admitting the coordinate system related with the homeomorphism $T$. The defined function is a vector of $C^{\infty}(G)$.

It is easy to see that the linear subspace $C^{\infty}(G)$ of the space $C(G)$ is the invariant space under the left and right translations. Furthermore, if $f \in C^{\infty}(G)$ then $\left.\frac{d}{d t} L_{h(t)} f(g)\right|_{t=0} \in C^{\infty}(G)$ and $\left.\frac{d}{d t} R_{h(t)} f(g)\right|_{t=0} \in C^{\infty}(G)$ for any one-parameter subgroup $h(t) \subset G$, where $L_{h}$ and $R_{h}$ are operators of the left and right translations respectively.

6.2. Let $\mathfrak{U}(G)$ be the linear space of all the almost periodic functions $f(g)$ on the topological group $G$. The intersection $\mathfrak{A}_{c}(G)=\mathfrak{A}(G) \cap C(G)$ is the linear subspace of the space $C(G)$.

Lemma. For the real properly nilpotent entire Lie group $G$ the linear space $\mathfrak{A}_{c}(G)$ is a non-trivial subspace of the space $C(G)$.

Proof. Let $H$ be the connected, finitely-dimensional Lie subgroup of $G$ defined in the point 5.7. The group $H$ has the non-trivial $\mathfrak{A}_{c}(H)$. The linear mapping $\varphi: C(H) \ni f_{H} \rightarrow \varphi\left(f_{H}\right)=f_{G} \in C(G)$ defined by $\varphi\left(f_{H}\right)(g)=f_{H, T}\left(t_{1}, \ldots, t_{n}\right)$ is continuous as $\left\|\varphi\left(f_{H}\right)\right\|_{G}=\sup \left\{\left|f_{G}(g) \|\right| g \in G\right\}$ $=\sup \left\{\left|f_{H}(h)\right| \mid h \in H\right\}=\left\|f_{H}\right\|_{H}$. Hence the image of every compact set $K_{H}$ is a precompact set $K_{G}=\varphi\left(K_{H}\right)$ in $C(G)$. Consequently the set $K_{G}=\left\{L_{g} f_{G} \mid g \in G\right\} \subset C(G)$ is precompact as $K_{G}$ is the image of the compact set $K_{H}=\left\{L_{h} f_{H} \mid h \in H\right\}, f_{H} \in \mathfrak{A}_{c}(H)$. This proves that the function $f_{G}$ is an almost periodic function on $G$. As $f_{G} \in C(G)$ it follows that $f_{G} \in \mathfrak{H}_{c}(G)$.

6.3. Lemma. For the real nilpotent entire Lie group $G$ the linear space $\mathfrak{A}_{c}(G)$ is a non-trivial subspace of the space $C(G)$. 
Proof. The real properly nilpotent entire Lie group $H=G / G_{n}$, defined also by $H=A_{n} G$ and $A_{n}$ is the mapping of the point 5.4, has the non-trivial $\mathfrak{U}_{c}(H)$ according to the Lemma 6.2. For every $f_{H} \in \mathfrak{U}_{c}(H)$ we define $f_{G}(g)=f_{H}\left(g_{H}\right)$ and $g_{H} \in H$ is the element of $H$ which is the image under the natural mapping $\psi: G \ni g \rightarrow \psi(g)=g_{H}=g \cdot G_{n} \in H$. Then the linear mapping $\varphi: C(H) \ni f_{H} \rightarrow \varphi\left(f_{H}\right)=f_{G} \in C(G)$ is continuous so that by the same argumentation as in 7.2 we conclude that $\mathfrak{U}_{c}(H) \ni f_{H} \rightarrow \varphi\left(f_{H}\right)=f_{G} \in \mathfrak{Q}_{c}(G)$.

6.4. We denote by $E$ the normed space with the norm $E \ni u \rightarrow\|u\| \in R_{+}$. Let $G \times E \ni(g, u) \rightarrow U(g) u \in E$ be a continuous representation of the topological group $G$ on the space $E$. The linear functional $M \in E^{*}$ will be called invariant if $M(U(g) u)=M(u), g \in G, u \in E$. The existence of such functionals follows from a simple consideration by Dixmier [6] of an equivalent problem for semigroups. Let $F$ be the subspace of the space $E$ spanned by all the vectors of the form $f=\sum_{k=1}^{n}\left(u_{k}-U\left(g_{k}\right) u_{k}\right)$, where $u_{1}, \ldots, u_{n}$, and $g_{1}, \ldots, g_{n}$, are any $n$ vectors from $E$ and any $n$ elements from $G$ respectively. Let us define $M(u)=0, u \in F$. There is a continuous non-trivial functional $M$ on the space $E$ which is a continuation of the functional $M$ defined by $M(u)=0$ on $F$. The acquired functional is invariant as $M(u)-M(U(g) u)=M(u-U(g) u)=0$.

Let $E=C(G)$ and $F$ a left and right translationally invariant subspace of $C(G)$ which contains the constant function on $G$. An invariant functional $M \in F^{*}$ for which $M(1)=1$ and $M(f) \geqq 0$ if $f(g) \geqq 0, f \in F$, is called a left invariant mean on $F$. Similarly one defines the right invariant mean and the invariant (two-sided) mean on $F$. The name is justified as the conditions are equivalent to

$$
\inf \{f(g) \mid g \in G\} \leqq M(f) \leqq \sup \{f(g) \mid g \in G\}, \quad f(g) \geqq 0 .
$$

There are groups for which no invariant mean exists on $C(G)$. However, for every $G$ the unique invariant mean exists on the subspace $\mathfrak{A}(G)$ of all the almost periodic functions on $G$ (von Neumann [20]).

Let $M$ be the invariant mean on $\mathfrak{A}_{c}(G)$. The function $\varphi(g)=M\left(\left(L_{g} \bar{f}\right) f\right)$ for every $f \in \mathfrak{A}_{c}(G)$ is a continuous positively definite function on the group $G$. The continuous positive definite functions on the topological group $G$ and the continuous unitary representations of $G$ are in one-toone correspondence (Gelfand and Raikov [9]). Hence the real properly nilpotent and nilpotent entire Lie groups $G$ with Lie algebra $X$ have continuous unitary representations according to the Lemmas 6.2 and 6.3. This shows that the next theorem has a sense.

6.5. Theorem. Let $G \times \mathfrak{H} \ni(g, u) \rightarrow U(g) u \in \mathfrak{H}$ be a continuous unitary representation of the real entire Lie group $G$ having Lie algebra $X$ on the 
separable Hilbert space $\mathfrak{H}$. Then $\mathfrak{H}$ is the direct sum $\sum_{k=1}^{\infty} \oplus \mathfrak{H}_{k}$ of the subspaces $\mathfrak{S}_{k}, k=1,2, \ldots$, each being the closure of the linear manifold $\mathfrak{D}_{k}$ where $\mathfrak{D}_{k}, k=1,2, \ldots$, are invariant under the representation of the group and invariant under the associated representation of the Lie algebra $X$ of $G$ into the Lie algebra of skew-symmetric operators on $\mathfrak{D}_{k}$.

Proof. The construction of $\mathfrak{D}_{k}, k=1,2, \ldots$, is in the full analogy with the construction of the Gårding's domain. Let us take $u, v \in \mathfrak{G}$ and let us consider the function $\tilde{f}(g, v, u, f)=f(g)(v, U(g) u)_{\mathfrak{H}}$, where $f(g) \in C^{\infty}(G)$. Because of the continuity of the representation $\tilde{f}(g, v, u, f) \in C(G)$ for a continuous left invariant functional $M$ on the space $C(G)$ we have $M(\tilde{f}(v, u, f))=M\left(L_{q} \tilde{f}(v, u, f)\right)$. The functional $\mathfrak{H} \times \mathfrak{H} \times C(G)$ $\rightarrow M(\tilde{f}(v, u, f)) \in C$ is antilinear in the first variable linear in the last two variables and simultaneously continuous in all three variables. Hence

$$
M(\tilde{f}(v, u, f))=(v, Q(f) u)_{\mathfrak{H}},
$$

where $Q(f)$ for a fixed $f \in C^{\infty}(G)$ is a bounded operator on $\mathfrak{H}$ with the norm not higher than $\kappa\|f\|_{G}, \kappa$ is a positive constant.

Now we define $\mathfrak{D}_{1}:=\left\{Q(f) u_{1} \mid f \in C^{\infty}(G)\right\}$, where $u_{1} \in \mathfrak{H}$ is a fixed vector. $\mathfrak{D}_{1}$ is a linear subspace of the space $\mathfrak{Y}$ as $Q\left(f_{1}+f_{2}\right)=Q\left(f_{1}\right)+Q\left(f_{2}\right)$ and $Q(\lambda f)=\lambda Q(f), f_{1}, f_{2}, f \in C^{\infty}(G), \lambda \in C$. If $\frac{\overline{\mathfrak{D}}_{1}=\mathfrak{H}_{1}}{n} \neq \mathfrak{H}$ we choose $u_{2} \in \mathfrak{S} \ominus \mathfrak{S}_{1}$ and proceed in construing $\mathfrak{D}_{2}$. If $\bigcup_{k=1} \mathfrak{D}_{k} \neq \mathfrak{H}$ for every $n$ then a sequence $\mathfrak{D}_{1}, \mathfrak{D}_{2}, \ldots$, exists with $\overline{\bigcup_{k=1}^{\infty} \mathfrak{D}_{k}}=\mathfrak{H}$.

The invarinace of $\mathfrak{D}_{k}$ under the representation of the group follows from the invariance of the functional $M$ as $M(\tilde{f}(v, u, f))=M\left(L_{g} \tilde{f}(v, u, f)\right)$ implies $(v, Q(f) u)_{\mathfrak{H}}=\left(v, U(g) Q\left(L_{g} f\right) u\right)_{\mathfrak{H}}$. This gives us

$$
U(g) Q(f)=Q\left(L_{g-1} f\right) .
$$

From the unitarity of the representation and our construction we have $\mathfrak{D}_{k} \perp \mathfrak{D}_{l}$ if $k \neq l$. Hence $\mathfrak{S}=\sum_{k=1}^{\infty} \oplus \mathfrak{S}_{k}$, where $\mathfrak{S}_{k}=\overline{\mathfrak{D}_{k}}$.

The vectors $t^{-1}\left(U\left(g_{x}(t)\right)-I\right) v$, where $g_{x}(t)$ is the one-parameter subgroup defined by $T\left(g_{x}(t)\right)=t x \in\{X, \gamma\}$, have the strong limes as $t \rightarrow 0$ for every $v \in \mathfrak{D}_{k}, k=1,2, \ldots$, and every one-parameter subgroup $g_{x}(t) \subset G$. The statement is the consequence of the continuity of the map- 
ping $C^{\infty}(G) \ni f \rightarrow Q(f) \in \mathfrak{Q}(\mathfrak{H}, \mathfrak{H})$ :

$$
\begin{gathered}
\left\|t^{-1}[U(g(t))-I] Q(f) u_{k}-Q\left(f_{x}\right) u_{k}\right\|_{\mathfrak{S}}=\| Q\left[\frac{1}{t}\left(L_{g_{x}(-t)} f-f\right)-f_{x}\right] \\
u_{k}\left\|_{\mathfrak{S}} \leqq \kappa\right\| \frac{1}{t}\left(L_{g_{x}(-t)} f-f\right)-f_{x}\left\|_{G}\right\| u_{k} \|_{\mathfrak{F}},
\end{gathered}
$$

where $f_{x}(h)=\left.\frac{d}{d t} L_{g_{x}(-t)} f(h)\right|_{t=0} \in C^{\infty}(G)$. We read that the element $x \in\{X, \gamma\}$ is represented by the skew-symmetric operator $d U(x)$ $=\left.\frac{d}{d t} U\left(g_{x}(t)\right)\right|_{t=0}$ on $\sum_{k=1}^{\infty} \mathfrak{D}_{k}$. The theorem is proved.

6.6. Let $\varrho:\{X, \gamma\} \ni x \rightarrow \varrho(x) \in \mathfrak{Q}\left(\mathfrak{D}_{k}, \mathfrak{D}_{k}\right)$ be the representation of the Theorem 6.5 and let us suppose that the vectors $u_{k} \in \mathfrak{D}_{k}$ are of norm $1:\left\|u_{k}\right\|_{\mathfrak{S}}=1, k=1,2, \ldots$ The functions $p_{f}: \mathfrak{E}\left(\mathfrak{D}_{k}, \mathfrak{D}_{k}\right) \ni \varrho(x) \rightarrow p_{f}(\varrho(x))$ $=\left\|f_{x}\right\|_{G} \in R_{+}$define the convex, positive functionals because of

$$
\begin{aligned}
f_{\lambda_{1} x_{1}+\lambda_{2} x_{2}}(g) & =\left.\frac{d}{d t} L_{g_{\lambda_{1} x_{1}+\lambda_{2} x_{2}}(-t)} f(g)\right|_{t=0} \\
& =\left.\frac{d}{d t} L_{g_{\lambda_{1} x_{1}}(-t)} L_{g_{\lambda_{2} x_{2}}(-t)} f(g)\right|_{t=0}=\lambda_{1} f_{x_{1}}(g)+\lambda_{2} f_{x_{2}}(g) .
\end{aligned}
$$

The vector space $\mathfrak{Q}\left(\mathfrak{D}_{k}, \mathfrak{D}_{k}\right)$. furnished with the topology defined by the family of seminorms $\mathrm{p}_{f}, f \in C^{\infty}(G)$, is the l.c. space $\mathfrak{I}_{G}\left(\mathfrak{D}_{k}, \mathfrak{D}_{k}\right)$ and in the same time the 1.c. Lie algebra if the mapping $L$ of 1.1 is defined as the bracket operation of two linear operators on $\mathfrak{D}_{k}$. The absolutely convex hull of $\mathfrak{L}_{G}\left(\mathfrak{D}_{k}, \mathfrak{D}_{k}\right), k=1,2, \ldots$, is denoted by $\mathfrak{L}_{G}(\mathfrak{D}, \mathfrak{D})$. It follows that $\mathfrak{I}_{G}(\mathfrak{D}, \mathfrak{D})$ is the 1.c. Lie algebra.

Lemma. The homomorphism $\varrho:\{X, \gamma\} \ni x \rightarrow \varrho(x) \in \mathfrak{L}_{G}(\mathfrak{D}, \mathfrak{D})$ is continuous.

Proof. It suffices to prove the continuity of $\varrho$ from $\{X, \gamma\}$ into $\mathfrak{I}_{G}\left(\mathfrak{D}_{k}, \mathfrak{D}_{k}\right)$ for every $k=1,2, \ldots$ The mapping $\{X, \gamma\} \times G \ni(x, g)$ $\left.\rightarrow \frac{d}{d t}(t x \circ T(g))\right|_{t=0}=\sum \frac{1}{(m+1) !}[\operatorname{ad} T(g)]^{m} x \in\{X, \gamma\}$ is continuous. The topological spaces $\{X, \gamma\}$ and $G$ are homeomorphic and, therefore, the continuity of $G \ni g \rightarrow f(g) \in C, f \in C^{\infty}(G)$, implies the statement of the Lemma.

6.7. Corollary. Real properly nilpotent and nilpotent l.c. Lie algebras $\{X, \gamma\}$ which are Lie algebras of real properly nilpotent and nilpotent Lie groups $G$ have continuous representations into the l.c. Lie algebra $\mathfrak{I}_{G}(\mathfrak{D}, \mathfrak{D})$ of skew-symmetric operators on the common invariant dense domain $\mathfrak{D}$ in a Hilbert space. 


\section{Remarks on Representations of Lie Field Structures}

7.1. We do not intend to enumerate the works in theoretical physics which deal with the infinite-dimensional 1.c. Lie algebras of any type, but only those works in which to our knowledge properly nilpotent or nilpotent 1.c. Lie algebras are considered. Bernstein and Halpern [3] obtained a Lie algebra of operators on a Hilbert space which is the sum of a nilpotent ideal and a semisimple finite-dimensional Lie algebra. As this algebra is already an algebra of operators on a Hilbert space we have not much to say about it.

7.2. More we can say about so called Lie field structures. A model of a quantum field theory which is formally a Lie algebra of operators on a Hilbert space was proposed by Greenberg [10]. The existence of Lie field structures as the l.c. Lie algebras regardless of its possible representation is proved by Lowenstein [15]. We do not know whether the LSZaxioms are satisfied for this model of quantum field theory but if they are the model gives only the trivial $S$-matrix in sense of LSZ formalism as is shown by Greenberg [11]. This question is still unanswered as the information of our note is still far from being sufficient to respond to this problem.

We can make a supplement to these considerations only concerning the existence of the representations of Lie field structures into the Lie algebra of symmetric operators on invariant common domain in a separable Hilbert space.

Let $Y$ be the linear space of functions $x(p): R_{4} \ni p \rightarrow x(p) \in C$ such that $Y$ is invariant under the representation of the Inhomogeneous Lorentz group I $L G \times Y \ni(\{\Lambda, a\}, x) \rightarrow U(\Lambda, a) x:=\left\{e^{i p a} x\left(\Lambda^{-1} p\right) \mid p \in R_{4}\right\} \in Y$. Let $\left\{x_{0}\right\}$ be a one-dimensional Lie algebra and let us consider the following composition rule for $X=Y+\left\{x_{0}\right\}$ :

$$
\begin{aligned}
& X \times X \ni(x, y) \rightarrow L(x, y): \\
& \quad\left\{x_{\theta} \int \Delta(p) x(p) y(-p) d p^{4}+\int c(p-q, q) x(p-q) y(q) d q^{4} \mid p \in R_{4}\right\} \in X,
\end{aligned}
$$

where $x_{0}$ is the central element of $X$. Under certain conditions on the functions $\Delta(p)$ and $c(p, q)$ (see for instance Lowenstein [1]) the mapping $L$ satisfies the conditions (1.2) so that $X$ becomes the abstract Lie algebra over the field of complex numbers. The algebra $X$ is the complexification $X=X^{c}$ of the real Lie algebra of all the functions $x(p), x \in Y$, for which $\overline{x(p)}=x(-p)$. We have an additional feature which indicates the nilpotency of Lie field structures. Let us denote $H_{m}=\left\{p \in R_{4} \mid p_{0}^{2}-\boldsymbol{p}^{2}>m^{2}, m>0\right\}$. Then $\operatorname{supp}\left(\int c(p-q) x(p-q) y(q) d q^{4}\right) \subset H_{m_{1}+m_{2}} \cap H_{\mu}$ if $\operatorname{supp}(x(p)) \subset H_{m_{1}}$ and $\operatorname{supp}(y(q)) \subset H_{m_{2}}$, where $\mu>0$ is the minimal mass of the theory.

7.3. An example of Lowenstein [1] shows that $c(p, q)$ may be a function bounded by 1 on $R_{4} \times R_{4}$. For such function $c(p, q)$ we can introduce 
a topology $\tau$ on the linear space $Y$. Let $K_{m}, m=1,2, \ldots$, be closed bounded balls of $R_{4}$ centered at the origin with radii $m$. We take a sequence of monotonically increasing functions $\varrho_{1}(p), \varrho_{2}(p), \ldots$, of the variable $p_{0}^{2}+p^{2}, p \in R_{4}$, where $\varrho_{1}(p)<\varrho_{2}(p)<\cdots$, such that $\varrho_{k-1}(p) / \varrho_{k}(p)$ tends to zero as $p \rightarrow \infty, k=1,2, \ldots$, and every $\varrho_{k}(p)$ fulfills the conditions: a) $\left.\varrho_{k}(p)\right|_{p=0}=1, k=1,2, \ldots$; b) $\varrho_{1}(p) \geqq|\Delta(p)|$ on every $K_{m_{1}} \cap H_{m_{2}}$; and c) $\varrho_{k}(p+q) \leqq \varrho_{k+1}(p)+\varrho_{k-1}(q)$. To satisfy this last condition it suffices to put $\ln \varrho_{k+1}(p) \geqq \ln \varrho_{k}(2 p)$. The metrizable 1.c. space $Y$ with the topology defined by the sequence of seminorms $Y \ni x \rightarrow p_{k}(x)=\int \varrho_{k}(p)|x(p)| d p^{4} \in R_{+}$ admits the 1.c. Lie algebra $\{X, \tau\}$ if we postulate $p_{k}\left(x_{0}\right)=1, k=1,2, \ldots$, because of

$$
p_{k}(L(x, y)) \leqq p_{1}(x) p_{1}(y)+p_{k+1}(x) p_{k+1}(y) \leqq 2 p_{k+1}(x) p_{k+1}(y) .
$$

The l.c. Lie algebra $\{X, \tau\}$ is the nilpotent 1.c. Lie algebra and for its real nilpotent entire Lie group of 7.2 we can apply the results of Section 6 . By the Corollary 6.7 we know that the nilpotent l.c. Lie algebra $\{X, \gamma\}$ with the topology $\gamma$ of the Theorems 5.8 has the continuous representations into the 1.c. Lie algebra of skew-symmetric operators on a common invariant domain of a Hilbert space.

7.4. The Lie field structure with the l.c. topology and the positive minimal mass allows the real nilpotent entire Lie group $G$ by the Theorem 5.8. If the answer to 5.9 is affirmative the inverse would be possible, i.e. the real nilpotent entire Lie groups with certain Lie groups of automorphisms (for instance the Inhomogeneous Lorentz group) would give the Lie field structures. In this case the real nilpotent entire Lie groups might be more convenient objects in searching for Lie field structures.

\section{References}

1. Balachandran, V. K.: On the uniqueness of the inner product topology in semi-simple $L^{*}$-algebra. Topology 7, 305 (1968).

2. Birkhoff, G.: Analytical groups. Trans. Am. Math. Soc. 43, 61 (1938).

3. Bernstein, H. J., and F. R. Halpern: Some consequences of an intrinsic mass formula. Phys. Rev. 151, 1226 (1966).

4. Bourbaki, N.: Eléments de mathématique, Livre V, Espaces vectoriels topologiques. Act. Sci. Ind. 1955, 1229.

5. Dieudonné, J., and L. Schwartz: La dualité dans les espaces $(F)$ et $(L F)$. Ann. Inst. Fourier 1, 61 (1950).

6. Dixmier, J.: Les moyennes invariantes dans les semi-groupes et leurs applications. Acta Sci. Math. Szeged 12, 213 (1950).

7. Dynkin, E. B.: Normed Lie algebras and analytic groups. Uspekhi Mat. Nauk 5, 135 (1950). See also Translations of Am. Math. Soc.

8. Gårding, L.: Note on continuous representations of Lie groups. Proc. Nat. Acad. Sci U.S. 33, 331 (1947). 
9. Gelfand, I. M., and D. A. Raikov: Continuous unitary representations of locally compact groups. Rec. Math-Math. Sbornik 13, 301 (1943). See also Translations of Am. Math. Soc.

10. Greenberg, O. W.: Generalized free fields and models of local field theory. Ann. Phys. 16, 158 (1961).

11. - Absence of interaction in Lie field theories. Commun. Math. Phys. 9, 13 (1968).

12. Grothendieck, A.: Produits tensoriels topologiques et espaces nucléaires. Mem. Am. Math. Soc. Nr. 16 (1955).

13. Hewitt, E., and K. A. Ross: Abstract harmonic analysis New York: Academic Press, Inc. 1963.

14. Köthe, G.: Topologische lineare Räume. Berlin-Göttingen-Heidelberg: Springer 1960.

15. Lowenstein, J. H.: The existence of scalar Lie fields. Commun. Math. Phys. 6, 49 (1967).

16. Pietsch, A.: Nukleare lokalkonvexe Räume. Berlin: Akademie-Verlag 1965.

17. Schue, J. R.: Hilbert space methods in the theory of Lie algebras. Trans. Am. Math. Soc. 95, 69 (1960).

18. - Cartan decomposition for $L^{*}$-algebras. Trans. Am. Math. Soc. 98, 334 (1961).

19. Veisfeiler, B. Yu.: Infinite-dimensional filtred Lie algebras and their connection with graded Lie algebras. Functional Analysis and its Applications 2, 88 (Engl.) or 94 (Russ.) (1968).

20. Neumann, J. von: Almost periodic function in a group I. Trans. Am. Math. Soc. 36, 445 (1934).

\author{
N. Limić \\ The Institute for Advanced Study, \\ Princeton, New Jersey 08540, USA
}

